\title{
Network Traffic Load Balancing In Gateways
}

\author{
${ }^{1}$ Jopxon P J, ${ }^{2}$ Arun Soman \\ ${ }^{l}$ Dept. of IT RSET Kochi, India \\ ${ }^{2}$ Asst. Professor, Dept. of IT RSET Kochi, India
}

\begin{abstract}
Load balancing is the practice of evenly distributing work among multiple devices. This technique provides several important benefits. Load Balancing in Gateways is a very important in today's emerging world. All the traffic of the computer network flows through the gateways of its network. A network gateway is an internetworking system capable of joining together two networks that use different base protocols. A network gateway can be implemented completely in software, completely in hardware, or as a combination of both. Network has great importance in gateway load balancing mechanism. The gateway node plays an important role as a bridge between different networks domains. The traffic load in gateways is a crucial part of the network which needs to be resolved and are important in order to reduce the load imbalance and to maximize the total network throughput. Such gateway load balancing is considered to improve the network performance more effectively than multipath load balancing. In this seminar, I am trying to show the network traffic, importance of traffic management in network especially in the gateways and point out that performing gateway load balancing is vital in order to take full advantage of the resources available and thereby improve the performance. A research study in this area and methodology to identify gateway load-balancing and characterize load-balanced paths is performed.
\end{abstract}

Keywords: GLBP,OSPF, Emulator, Interface tracking, Network optimization.

\section{Introduction}

Load balancing is designed to provide high availability and high reliability, as well as high scalability. Load balancing is a computer networking method or technology for distributing the workloads in the network between multiple paths available or across the entire network in most balanced way. The practice of load balancing in the network will allow the optimization of resources it will reduce the response time of the network and will help to reduce the network overload [1]. In normal scenario the load balancing protocols will consider only a single path for the purpose of load balancing in network. If a new system that helps considering multiple paths for load balancing then it can improve the load balancing mechanism in network. The use of multiple path or multiple gateways at the same time in network will help us to balance or distribute the entire load of the network intelligently. The ultimate aim of load balancing in network is to provide high availability in network [3]. In order to achieve high availability a protocol that has the capacity to use multiple paths at the same time needs to be used. Gateway load balancing protocol is one of the available protocols that have the capability to consider multiple path at the same time. So the protocol GLBP is used for the research study. There are other protocols available like HSRP and VRRP but it can consider only one gateway at a time. Network always continues to grow. The day to day use of the network continues to grow so appropriate load balancing scheme or mechanism must be implemented otherwise the entire network may become unstable and it can cause a huge loss in terms of service levels. The analysis study is to find best solution to the problem of load balancing in network. If suppose one of the active path that connects the network is disconnected or connection is lost it will definitely produce loss of data and it will affect the service percentage. So at the real time a new mechanism must be implemented that can avoid the data loss. The work carried out is to find a best way to these types of issues in network. If redundant path are available in network it can be used and the traffic can be forwarded to those available path [2]. This can help to achieve good networking. The gateway load balancing protocol can enhance redundancy and can increase the performance of the network by fully utilizing the available network resources. Through this method the single point of failure can be removed. If using gateway load balancing protocol efficient use network resources. If multiple paths are available it allows using those available paths for the transfer of data in network without data loss. It allows the routers to choose another gateway if current gateway is fails [4]. As it can automatically detect the failure of any network connection and reroute to another available path the administration cost can be reduced. This is one of the best features of this protocol. It allows us to send the traffic packets to any hosts on any other networks with balancing their traffic among multiple gateways. The load balancing mechanism can be used for good load balancing in network with the provision of giving weighting parameter [5].The GLBP protocol has the mechanism to consider multiple path at the same time unlike other available protocols. It provides a new idea that allows all the available gateway to participate in the packet forwarding election. The network load can be balanced across all the links without the use of 
complex protocols helps the network to achieve minimum overhead and minimum effort [8]. Network redundancy is the most important goal of the load balancing mechanism. The load of the network need to be analyzed dynamically and best effort scheme of routing the traffic is very essential for a balanced network. Network optimization must be achieved. The work performed is divided in to two section. First the configuration part of the topology and next shows the verification and results. The results of the work clearly shows the advantage of load balancing in the gateways of the network and hence the network can be optimized.

\section{Work carried out}

The topology created for the analysis and study is shown in figure (1). The network topology consists of six different networks. The router R4 and router R5 are the source and the destination is assigned to be router R1. There are two different routes through which the destination R1 router can be reached [7]. The study is to find the best possible path from the available routes and to route the packets to the best path to the destination. In the topology which is created there are two different paths available. First path connecting switch 1, router R2 and R1 another path is through route switch 1, router R3 and R1. For both host router R4 and router R5 which are designed as the source has connection to the switch SW1. The entire network and all the interfaces of the network are configured with IP addresses. For the purpose of the routing between the networks a routing protocol is essential. The routing protocol used for the work is open shortest path first protocol called as OSPF. The routing protocol must be configured at routers R1, R2 and R3. The aim of the work is to find a path to the destination from all the available paths. The next part of the work concentrates on finding a path to the destination if the current path is lost or shutdown. For that purpose a protocol called gateway load balancing protocol (GLBP) is used and configured in the network. The gateway load balancing protocol are configured at router R2 and router R3. At the source routers R4 and R5 the default gateway is configured and set. The connection establishment between all the nodes in the network are checked with the help of ping test process. Once the connection has been made and the communication between all the routers are established then the network is ready for the analysis and study. The router R4 and router R5 are configured with a default gateway. At the destination router R1 a loopback is configured for the purpose of testing the connection establishment and communication check between the source and the destination [6]. The gateway load balancing protocol needs to be configured and are configured between router R2 and router R3. The gateway is also configured with one particular IP address. In order to perform the load balancing based on considering the condition of the host it is configured accordingly. Another factor is regarding the priority assignment for routers. Based on the priority value the proper router can be selected for the load balancing according to the best possible way. Once the GLBP is configured the events can be verified and analyzed within the routers. If many different paths along with routers are available the priority value for each router can be assigned manually up on our wish. A method called the interface tracking can be configured and are configured on the interfaces of the routers. By doing so the nature of the interface configured with interface tracking can be analyzed [10]. A weight tracking method is also used in the work for understanding the load balancing scheme in the network. If suppose one of the important line or path gets down or failed then the weight assigned to them gets decreased. The weight tracking configured at the router will check for the change in its value. If it finds any link failure the weight assigned to them gets reduced. The important concept used here is that if the weight get decreased or reduced below a particular value then it can be configured like do not transfer or forward any more packet through that link [9]. So it will automatically check for other available path to forward the packets to the destination. Thus by using this new concept the load balancing can be controlled in a most suitable manner.

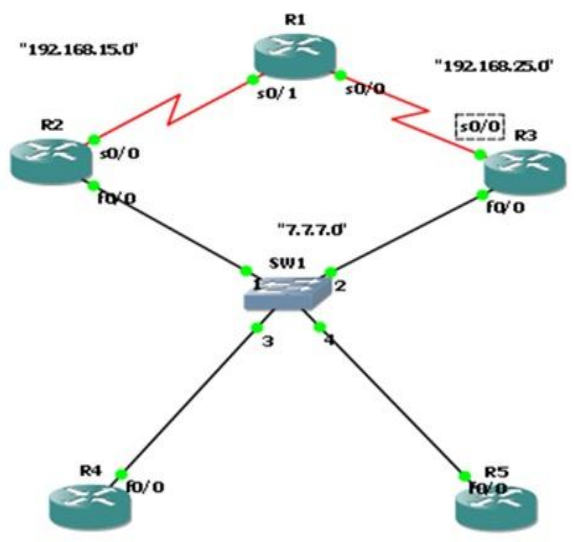

Fig. 1 Network Topology 


\section{Load balancing mechanism}

The next part of the work is about the analysis and research study regarding the load balancing mechanism. After performing all the required configuration at all the routers and checking the communication between the devices the result can be studied and analyzed [3]. In order to test the result of the work carried out the interface to the destination router R1 is intentionally closed or disconnected [7]. In the figure as it can been seen there are two path available to the destination router R1. First path is through SW1, R2 and R1 and the other path is SW1, R3 and R1. A communication is made from router R4 to router R1. The communication was verified at the router and analyzed that the communication happens through the path SW1, R3 and reaching router R1. To verify the analysis study the interface of router R1 that is serial interface $0 / 0$ connecting to the router R3 is disconnected. As the connection got disconnected the packets to the destination router R1 will be lost. In order to secure the traffic the packets are reassigned to flow through the next available path that is through the path SW1,R2 and reaching router R1 [6].

\section{Results}

In order to find the benefits of proper load balancing using the protocols a scenario is implemented using the help of the emulator. The output of the emulation clearly shows the importance of load balancing in gateways. Figure two shows the output verification of the work. If suppose a heavy load reaches the gateway it needs to be managed efficiently if not it can cause huge problems and can destroy the whole network. Gateways are the node or path through which the traffic flows

to other networks if the load is not maintained properly it creates large problems. In order to balance the load over the -

Table I Verification Results.

\begin{tabular}{|l|l|l|l|}
\hline Interface & State & Action & Result \\
\hline $\mathrm{R} 1-\mathrm{s} 0 / 0$ & Active & $\mathrm{ON}$ & 1 \\
\hline $\mathrm{R} 2-\mathrm{s} 0 / 1$ & Listen & OFF & 0 \\
\hline $\mathrm{R} 1-\mathrm{s} 0 / 0$ & Listen & OFF & 0 \\
\hline $\mathrm{R} 2-\mathrm{s} 0 / 1$ & Active & $\mathrm{ON}$ & 1 \\
\hline $\mathrm{R} 1-\mathrm{s} 0 / 0$ & Active & $\mathrm{ON}$ & 1 \\
\hline $\mathrm{R} 2-\mathrm{s} 0 / 1$ & Listen & OFF & 0 \\
\hline
\end{tabular}

available path we configure a special kind of protocol called gateway load balancing protocol. Using the help of this protocol we can efficiently manage the traffic load in the gateways. The important factor about this protocol is that if suppose a path through which load flows gets destroyed due to any of the reason the load will be automatically transferred to the next best available path.

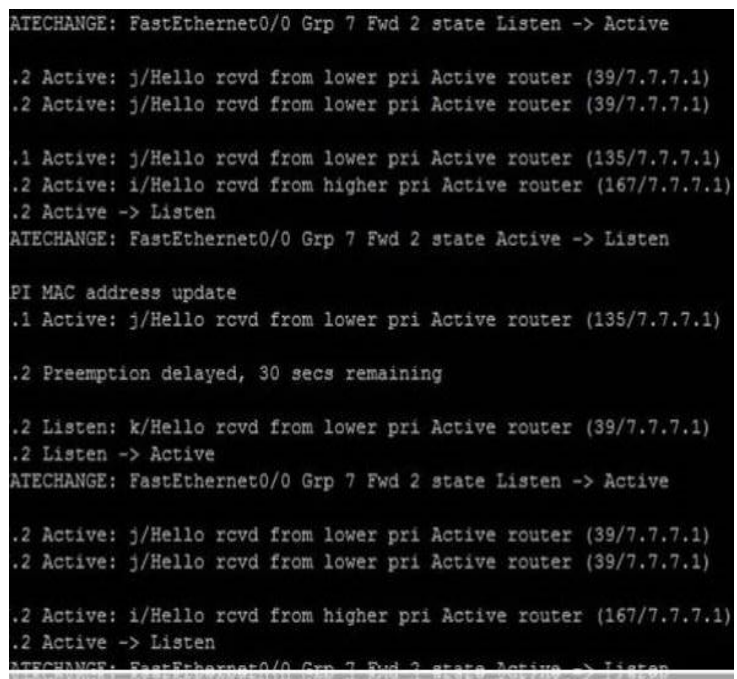

Fig. 2 Emulator Result.

We can also set the threshold values to all the available path. If the threshold value changes from its benchmark we can set the constrains according to the change. If the value gets decreased from the benchmark value we will set the GLBP configuration to find another path to forward the traffic to other networks similarly if the value of a particular path gets increased or comes back to the normal value we can set the constrains to 
make it as the active path to forward the traffic on the next chance. In the figure 1 see the topology configuring the load balancing parameters. The aim is to send traffic from router R4 and R5 to the destination router R1. For this we can send the traffic either through R4/R5-SW1- R2-R1 or through the path R4/R5-SW1-R3- R1. The features of the GLBP allows us to select anyone of the path to forward the traffic to the destination. If one available path fails the other path will get active automatically. The table 1 shows the vital changes in the topology. The action field of the table clearly shows the states on and off of the network. Similarly the result field of the table shows the values 1 and 0 where 1 means the interface is active and the value 0 shows the interface is down. To verify the changes in the network and to analyze the load balancing mechanism the serial interface $0 / 0$ of router R3 which is active is disconnected. The other path through router R2 with interface serial $0 / 1$ is in the listen state. But when the path through router R3 is disconnected then the packets will be reassigned to flow through the path through router R2. Now the router R2 interface serial 0/1 which was in listen state becomes active and the router $\mathrm{r} 3$ which was in active state becomes listen. This is the important action takes place for the load balancing mechanism. If the interface serial $0 / 0$ again becomes active it will allocate the traffic flow on the next change in the network

\section{Conclusion}

In this paper, the importance of traffic management in network especially in the gateways are performed and it points out that performing gateway load balancing is vital in order to take full advantage of the resources available and good performance. A research study in this area and methodology to identify gateway load balancing and characterize load-balanced paths is performed. At the present situation the protocols which are used for routing and load balancing are proprietary like juniper proprietary protocols, Cisco proprietary protocols. Open standard protocols for load balancing need to be developed. More and more research studies need to be performed in this field. The new method proposed can effectively balance the load in the network. The most important about the load balancing scheme is to bring high availability. In network the traffic must be given much priority. The packet loss cannot be acceptable. In order to make the traffic flow effective all the available path in the network must be considered and it must make use of those available route. The aim of the work is to distribute the load of the network evenly among all the available nodes and make the network more stable.

\section{Acknowledgment}

I extend my sincere and heartfelt thanks to Mr. Arun Soman, Asst. Professor, Department of Information Technology of RSET, for helping me in my presentation and providing with timely advices and guidance. I thank God almighty for all the blessing received during this endeavor. Last, but not least I thank my father and all my friends for the support and encouragement they have given me during the course of my work.

\section{References}

[1]. Yukitaka Yoshida and Masatoshi Kawarasaki, "Relay-Node Based Proactive Load Balancing Method in MPLS Network with Service Differentiation", Third IEEE Workshop on User-Centric Networking, 2012.

[2]. Camellia Askarian and Hamid Beigy, "A Survey for Load Balancing in Mobile WiMAX Networks",Advanced Computing: An International Journal, Vol.3, No.2, March 2012.

[3]. Chengjie Gu, Shunyi Zhuang, Yanfei Sun, Chao Liu and Nan Sun "Pricing Incentive Mechanism Supporting Load Balancing for QoS-Enabled Networks ,'July 31 2008-Aug. 12008.

[4]. Cisco papers “Quality of Service Networking,"August 26, 2012.

[5]. Cisco Network Academy Explorations, CCNA Guides.

[6]. Aaron Balchunas, "Redundancy and Load Balancing v1.21," 2009 August.

[7]. Todd Lammle, "CCNA Cisco Certified Network Associate: Study Guide ," ISBN-13 9788126531257, April 2011

[8]. Xiuzhong Chen, Chunfeng Wang, Dong Xuan, Zhongcheng Li, Yinghua Min, and Wei Zhao, "Survey on QoS Management of VoIP," March 2010.

[9]. Aaron Balchunas,CCNA Study Guide December 2011

[10]. Mathias Hein, David Griffiths, Orna Berry, “ Quality of service pricing network architecture supporting load balancing ," July 31 2008-Aug 1 2008,ISBN-978-1-4244-1866-4. 